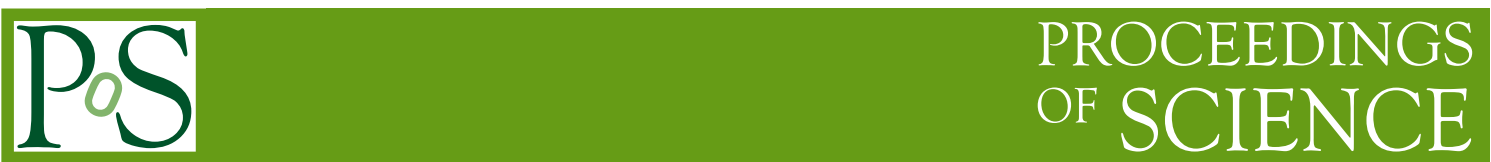

\title{
Performance of the ALICE silicon detectors
}

\author{
R. Santoro* \\ Dipartimento di Fisica dell'Università, Bari (Italy) and \\ CERN, European Organization for Nuclear Research, Geneva (Switzerland) \\ E-mail: romualdo.santoro@cern.ch \\ for the ITS Collaboration
}

The Inner Tracking System (ITS) is the central barrel detector closest to the interaction point of the ALICE experiment at LHC. It is a six-layers silicon detector installed around the beam pipe and it is built using three different technologies: the first two layers are equipped with pixel detectors (SPD), the two intermediate layers with drift detectors (SDD) and the last two layers with double-sided micro-strip detectors (SSD). The radius, the granularity and the technology of each layer were chosen in order to achieve excellent tracking, vertexing and particle identification capabilities in the high density particle environment expected in the central $\mathrm{Pb}-\mathrm{Pb}$ collisions at the LHC energy.

The detector performance after two years of experience with LHC proton and lead beams, as well as cosmic rays, will be overviewed in this paper.

The 20th Anniversary International Workshop on Vertex Detectors - VERTEX 2011

June 19 - 24, 2011

Rust, Lake Neusiedl, Austria

\footnotetext{
* Speaker.
} 


\section{Introduction}

ALICE $[1,2]$ is the heavy ion experiment at LHC devoted to study the strongly interacting matter under extreme conditions of high temperature and density as those achieved in ultra-relativistic $\mathrm{Pb}-\mathrm{Pb}$ collisions. The physics program includes also $\mathrm{p}-\mathrm{p}$ and $\mathrm{p}-\mathrm{A}$ collisions for comparison with respect to $\mathrm{Pb}-\mathrm{Pb}$ collisions. ALICE contributes to the $\mathrm{p}$-p physics program also with some genuine physics thanks to the low momentum cutoff (down to $100 \mathrm{MeV} / \mathrm{c}$ ), the excellent particle identification and the efficient minimum bias trigger.

The ALICE central barrel detector is designed to track and identify charged particles in a wide transverse momentum range $(100 \mathrm{MeV} / \mathrm{c}-100 \mathrm{GeV} / \mathrm{c})$ in the high density track environment normally produced with central $\mathrm{Pb}-\mathrm{Pb}$ collisions. It is mainly made of the Inner Tracking System (ITS), six-layers of silicon detectors with three different technology, and the Time-Projection Chamber (TPC); both embedded in a moderate magnetic field up to $0.5 \mathrm{~T}$. The two detectors together provide more than 150 hits per track in the central region $(|\eta| \leq 0.9)$ where the overall material budget is limited to $13 \% \mathrm{X} / \mathrm{X}_{0}$. These features allow a robust and reliable tracking with a low momentum cutoff down to $\approx 100 \mathrm{MeV}$, very good momentum resolution at high pt and excellent particle identification performance over a wide momentum range.

The position and segmentation of the six cylindrical layers of the ITS were designed to limit the occupancy to a few $\%$ assuming a particle density of 8000 in the central pseudorapidity unit. The two closest layers to the beam line are equipped with pixel detectors (SPD) and are placed at mean radii of 3.9 and $7.6 \mathrm{~cm}$, respectively. They contain about $10 \mathrm{M}$ pixel channels of dimensions $50 \mathrm{x}$ $425 \mu \mathrm{m}^{2}$ each. The pixel modules are mounted on a light carbon fiber structure and the total material budget per pixel layer is $1.14 \%$ of $\mathrm{X}_{0}$. The precise spatial information at only $3.9 \mathrm{~cm}$ from the beam line and the reduced material budget play a key role to determine the tracking pointing resolution. The two intermediate layers are equipped with silicon drift detectors (SDD) and are placed at mean radii of 15.0 and $23.9 \mathrm{~cm}$. This was a compromise to have precise bi-dimensional points with a reduced number of channels. The last two layers are equipped with double sided micro-strip detectors (SSD) at mean radii of 38.0 and $43.0 \mathrm{~cm}$. The larger distance from the interaction point guarantees a low occupancy (1 particle $/ \mathrm{cm}^{2}$ ) necessary to prevent a large hit ambiguity. The last SSD plane was placed reasonably close to the TPC in order to achieve an effective track matching between ITS and TPC.

The main ITS contributions to the ALICE detector performance are: the primary vertex reconstruction, the improvement of the impact parameter with a precision of $60 \mu \mathrm{m}$ at $1 \mathrm{GeV} / \mathrm{c}$ to detect the secondary vertices produced by the decays of hyperons and D and B mesons, the improvement of momentum resolution for particles reaching the TPC and also the standalone tracking and PID capability for low momentum particles. For this purpose, four out of six layers, namely SDD and $\mathrm{SSD}$, provide the analogue information which allows the particle identification via $\mathrm{dE} / \mathrm{dx}$ measurement in the non-relativistic $\left(1 / \beta^{2}\right)$ region.

The ITS has also the possibility to contribute to the ALICE L0 trigger decision with the first two layers. In fact, the SPD data stream includes a digital signal (Fast-OR) promptly asserted by each of the 1200 front-end chips which is elaborated according to predefined algorithms; the outcome signal is shipped to the Central Trigger Processor (CTP) in $\approx 800 \mathrm{nsec}$ [3]. This feature was extremely useful since the first commissioning of the SPD, the ALICE cosmic data-taking and the 
p-p collision runs[4]. More details on the ITS can be found in reference [2], hereafter an overview on the detector operation and performance will be discussed.

\section{ITS commissioning and operations}

Each sub-detector constituting the ITS has its own calibration procedures. At the beginning, systematic calibration studies have been performed to operate the detectors and to optimize their performance. Long runs, triggering on cosmic muons, have been carried out for the commissioning of the overall system and to verify its stability, to tune the $\mathrm{dE} / \mathrm{dx}$ information (SDD and SSD) and to align it $[5,6]$. At present, the series of calibration runs which are used to monitor the performance and to store the calibration data in the Off-line Condition Data Base (OCDB) are fully integrated in the ALICE framework. In fact, the shift crew running ALICE takes care of all calibrations on the basis of dedicated runs performed with the proper frequency (i.e. each day, each beam fill or on demand). At the end of the calibration runs, on-line Detector Algorithms (DAs) spot channel misbehaviors (i.e. noisy or dead) or extract the calibration parameters which are stored in the OCDB; information needed for the data reconstruction.

\subsection{SPD}

The SPD response has been optimized tuning several 8-bit DACs integrated in the Front-End Electronics (FEE). The configuration files, obtained during the module qualification tests, were used as starting point for the commissioning in the experimental area. A further optimization of the detector response in the ALICE environment was performed by tuning the most relevant DACs. The power consumption was also optimized to account for the reduced cooling efficiency of few specific sectors, which was experienced after the installation in the experimental area [7].

At regime, the main types of calibrations runs are the following:

- The internal pulser run, which injects a calibrated charge in the pre-amplifier and verifies the uniformity of the detector response. It is usually performed during each LHC technical stop ( $\approx 6$ weeks).

- The random-trigger run, which identifies the noisy pixels to be masked in the FEE and which is performed on demand. The detector response looks quite stable in time and even if a noisy pixel appears during the data-taking, the on-line algorithm automatically identifies and excludes it from the data-analysis. The same procedure identifies dead pixels $(\approx 0.6 \%)$ and pixels with efficiency lower than $40 \%(\approx 1.2 \%)$. Both categories are excluded from the off-line reconstruction.

- The SPD provides in parallel ten L0 trigger signals defined by ten corresponding algorithms. As mentioned above, each algorithm combines the 1200 Fast-OR signals ( 1 / chip) which are streamed out from the detector. One Fast-OR signal is active if at least one pixel in the chip matrix goes over threshold. The reliability of this signal is based on the absence of noisy pixels and on the efficient Fast-OR circuitry response which can be optimized tuning four dedicated DACs [8]. An additional calibration run is needed to optimize the trigger response. It is performed on each LHC technical stop since it was shown to be very stable in time. 
At the moment almost $30 \%$ of SPD modules cannot be stably operated due to the aforementioned cooling performance. There is a big effort to identify actions capable to recuperate the maximum number of modules even considering options that might require an intervention on the detector services.

\subsection{SDD}

The SDD commissioning was based on dedicated runs devoted to optimize the detector functioning and on the analysis of the data collected during the long sessions of cosmic data-taking [6]. During the normal detector operation, the calibration procedures are based on three types of runs performed during the LHC fills or at least once per day.

- The first procedure is the pedestal run which measures the baseline signal and identifies the noisy channels. At present, the baseline has been proven to be very stable at $\approx 2.3$ ADCs (i.e. a MIP signals is around 100 ADCs) with a low percentage of noisy channels $(\approx 0.7 \%)$. The baseline is then stored in the FEE to equalize the signals.

- The second procedure is the pulser run. A signal is applied on each pre-amplifier channel to measure the gain and to spot dead channels $(\approx 1.5 \%)$. This information is used to equalize the anode signal thus improving the charge balance and therefore the spatial reconstruction. An uniformity of around $4 \%$ has been measured along all the channels.

- The last procedure is the injector run. During this run a charge is injected in 33 positions along the anode coordinate of each sensor $(r \phi)$, at three different distances from the anode. This run is used to map the drift speed in the sensor since it has a big impact on the spatial accuracy of the coordinate measured by the drift time. The two main contributions to the drift speed are the temperature $\left(v_{\text {drift }} \propto T^{-2.4}\right)$ and the non uniformity of the drift field (i.e. non-linearities in the voltage divider or the inhomogeneities in dopant concentration). While the latter has been mapped in the laboratory with laser measurements [9], the former needs continues measurements. The accurate knowledge of the drift speed of $\approx 0.1 \%$ is important to reach the $35 \mu \mathrm{m}$ spatial resolution. The outcome of these measurements are stored in the OCDB and are used in the off-line reconstruction.

At present only 16 out of $260(\approx 6 \%)$ basic modules are out of acquisition. Six modules present high voltages problems (i.e. shorts or high leakage currents) while the others show problems with the on-board electronics.

\subsection{SSD}

The commissioning phase with cosmic tracks performed in 2008-2009 was used to align the detector and to calibrate the ADCs response, proportional to the charge released by the particles, and the charge matching for the signal released on the $\mathrm{P}$ - and $\mathrm{N}$ - side. This last calibration is important to solve the hit ambiguity.

During the normal operation, standalone calibration runs are performed at each beam fill using random triggers to measure the baseline signal and to tag noisy and dead channels. A typical value 

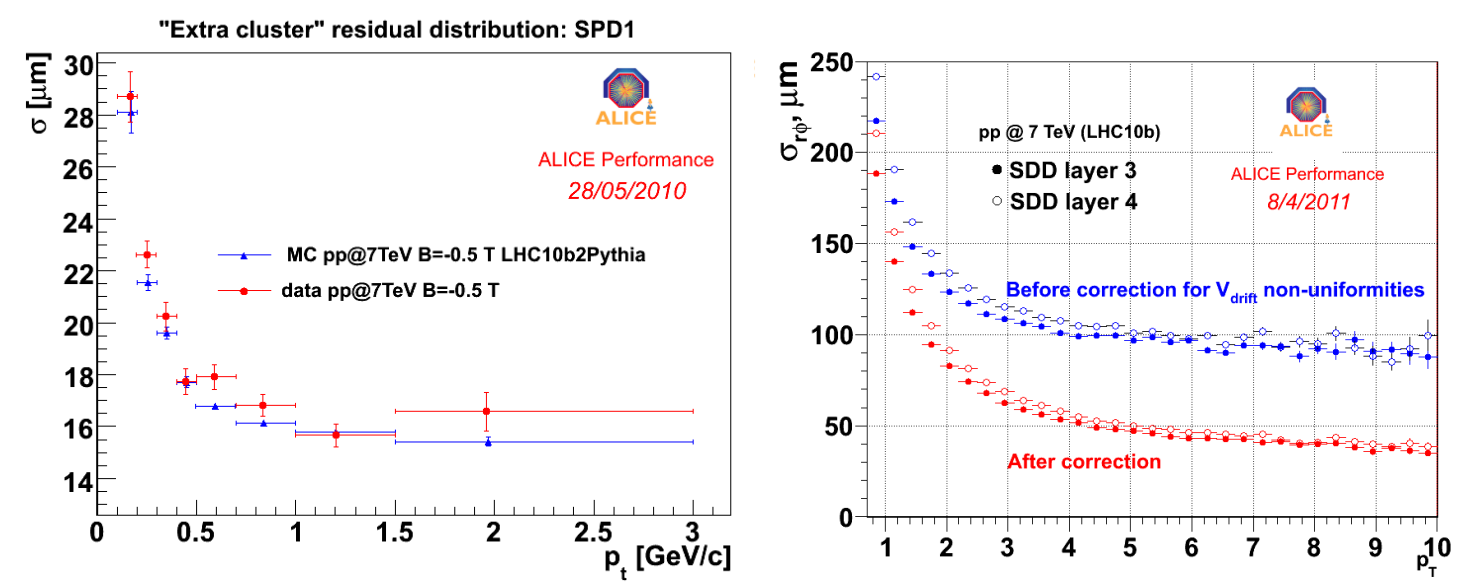

Figure 1: Examples of performance plots for the alignment: the $\sigma$ from residuals Gaussian fit measured in the overlap regions for the innermost SPD layer (left) and the $\sigma$ of track-to-point residuals in the bending plane before and after the $v_{\text {drift }}$ correction for the two SDD layers (right).

of 2.4 and 3.5 ADCs is measured for $\mathrm{P}$ and $\mathrm{N}$ side respectively with a signal-to-noise ratio bigger than 40 . The measured value is stored in the read-out electronics and it is used to adjust the signal or to mask on-line the noisy channels. The result of these runs are also stored in the OCDB to be used in the reconstruction.

At present, 141 modules out of $1698(\approx 8 \%)$ are not used in acquisition due to electrical or configuration problems. The modules in acquisition have good stability in time and only $1.5 \%$ of channels are excluded because noisy or dead.

\section{ITS performance with $\mathrm{Pb}-\mathrm{Pb}$ and $\mathrm{p}-\mathrm{p}$ data}

The ITS was installed in ALICE in June 2007. The services connection was completed in December 2007 and then the commissioning in the experimental area could start. Since the beginning of 2008, the commissioning with cosmic rays was carried on and in summer the full ITS was able to collect already more than $100 \mathrm{~K}$ of cosmic tracks, which were used for the sub-system response calibration and alignment. Since then, the ITS was fully integrated in ALICE and participated continuously in the experiment data taking. In November 2009 there was the first p-p commissioning run at $900 \mathrm{GeV}$, which was used by ALICE to measure the charge particle density at midrapidity in p-p collisions at $\sqrt{s}=900 \mathrm{GeV}$ [10]. In this phase, the SPD played a crucial role providing the minimum bias trigger signal. The analysis was entirely based on the ITS data. The ALICE data taking continued in 2010 and 2011 recording data during p-p collisions up to $7 \mathrm{TeV}$ and $\mathrm{Pb}-\mathrm{Pb}$ collisions at $\sqrt{s_{N N}}=2.76 \mathrm{TeV}$.

\subsection{Alignment}

In order to achieve the demanding requirements in terms of tracking efficiency and impact parameter resolution, a very accurate alignment and description of the material budget are fundamental. The ITS is made of 2198 individual sensor elements that have to be aligned in space. The 

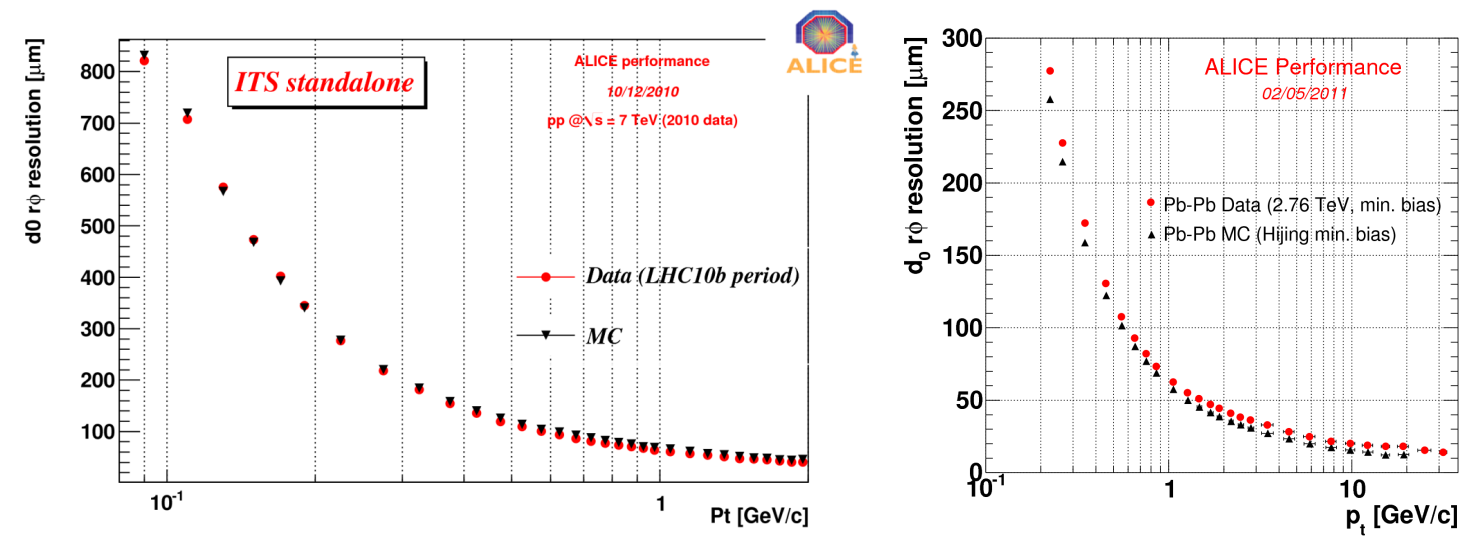

Figure 2: Transverse impact parameters in rphi for data and Montecarlo in the case of p-p beams at $7 \mathrm{TeV}$ and $\mathrm{Pb}-\mathrm{Pb}$ beams at $2.76 \mathrm{TeV}$.

data sample used for the preliminary alignment was the cosmic ray tracks collected in 2008 [11]. In 2010 a sample of p-p data was collected without magnetic field in order to refine the alignment in particular for the side elements of the ITS barrel. In fact, due to the ITS acceptance for mostly vertical cosmic rays, the sample of tracks traversing the side part of the barrel was very poor. Samples of $\mathrm{p}-\mathrm{p}$ data with magnetic field are collected periodically to monitor the alignment stability.

Two independent track-based alignment methods have been adopted: a global fit method named Millepede (default method) and a local fit method based on iterative residuals minimization [12]. The general strategy uses the survey measurements done for the SDD and SSD as alignment starting point. The cosmic ray tracks are firstly reconstructed using the SPD and SSD. The SDD, requiring a more elaborated calibration procedure due to the interplay between alignment, drift velocity and time-zero calibration, is added in a second step once a satisfactory alignment of the SPD and SSD is reached. The last step of the alignment procedure is the alignment of the whole ITS with respect to the TPC.

The figure of merit of the alignment is based on the track to point residuals for extra-clusters, i.e. the clusters placed in the layer overlap regions, or the track to point residuals extrapolated onto the layer which is not used in the tracking. An example of these two plots are shown in figure 1. The plot on the left shows the $\sigma$ of the track-to-point residuals obtained for the extra-cluster in the innermost SPD layer as a function of $p_{t}$ for p-p data at $7 \mathrm{TeV}$ and Montecarlo. The good matching between data and Montecarlo and the $p_{t}$ dependence are clearly visible in the figure. In fact, at high $p_{t}$ the main contributions to the $\sigma$ are the spatial precision and the residual misalignments, while at low $p_{t}$ the material budget contribution starts to have a bigger impact. The plot on the right shows the $\sigma$ of the Gaussian fit done on the track-to-point residuals for the two SDD layers. The improvement of the residuals after the correction for the non uniformity of the drift field along the drift path is clearly visible. The correction is extracted for each module starting from the measured residuals as a function of the drift distance. The result is used in the reconstruction to correct the raw drift coordinate in order to get an unbiased position of the particle crossing point. These correction maps were first measured during the construction phase with a laser [9] and are updated during the calibration phase of each data-taking period by using the track-to-point residuals from a 

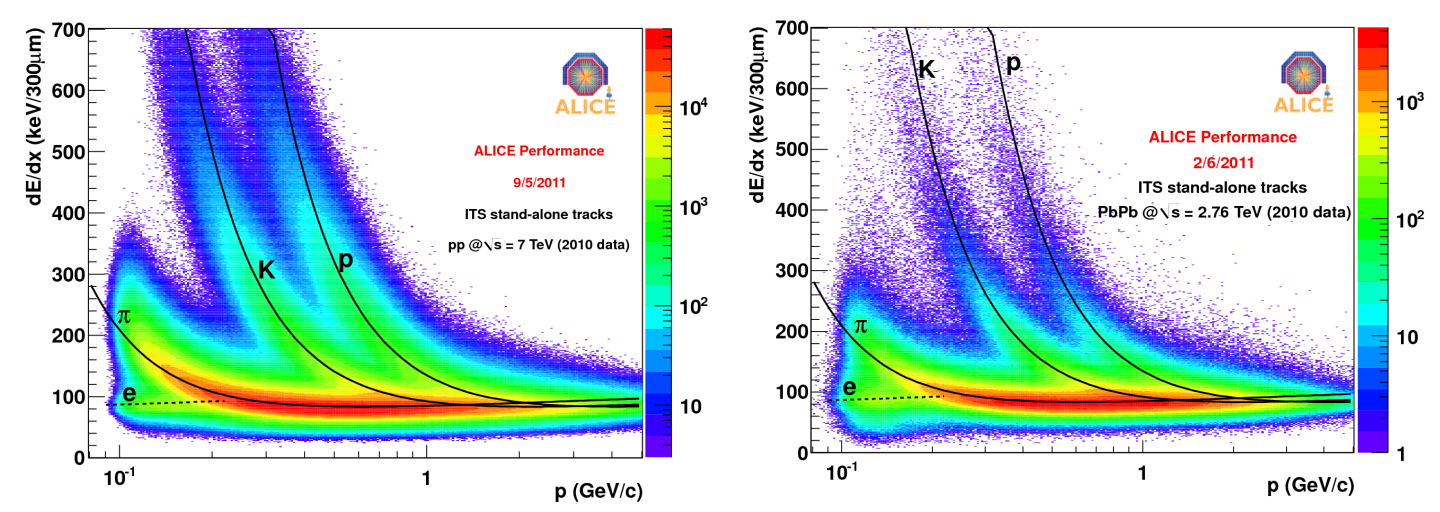

Figure 3: $\mathrm{dE} / \mathrm{dx}$ measurement of charged particles for the ITS standalone tracking in p-p at $7 \mathrm{TeV}$ (left) and $\mathrm{Pb}-\mathrm{Pb}$ at $2.76 \mathrm{TeV}$ (right) as a function of $p_{t}$.

subsample of reconstructed particles in the ITS.

\subsection{Tracking and PID capability}

The tracking is based on the Kalman filter [13]. The track reconstruction starts from the external part of the TPC and proceeds towards the innermost layer of the SPD. The TPC and ITS clusters are associated to a track following an iterative method which exploits the vertex reconstructed using only the combination of the clusters (tracklets) of the two SPD layers as a seed for the cluster matching. In each step of the cluster association, the track parameters are recalculated and a search window is opened to look for the next step. The search windows is tuned according to the hit density, the track extrapolation length and the residual misalignment. Once all the clusters are associated to the track, it is refitted outwards looking for the informations provided by the outer detectors in the barrel (i.e. TOF, TRD), using in addition the particle identification capability. This last step is particularly useful in central $\mathrm{Pb}-\mathrm{Pb}$ events. Once the clusters associated to the combined TPC-ITS tracks are picked up from the reconstructed event, the stand-alone tracking procedure starts with the aim of finding tracks that do not reach the TPC. The ITS stand-alone tracking procedure starts from the main vertex towards the last SSD layer. In this algorithm, the searching windows are progressively enlarged to search for clusters originated by low $p_{t}$ particles. Only after that the clusters belonging to the high momentum tracks are removed (i.e. almost straight line at small radii) and the hit multiplicity is strongly reduced, the search windows can be efficiently enlarged [14].

The very low material budget in the ITS and the short distance between the first layer and the interaction point are crucial parameters to obtain good tracking performance at very low $p_{t}$. In figure 2 we show the transverse impact parameter in the bending plane for data and Montecarlo in the case of p-p at $7 \mathrm{TeV}$ and $\mathrm{Pb}-\mathrm{Pb}$ at $2.76 \mathrm{TeV}$ from the ITS stand-alone tracking. Thanks to the precise alignment and the low material budget, data and Montecarlo agree within 10\%. The impact parameter resolution for particles with momentum greater than $1 \mathrm{GeV}$ is better than $65 \mu \mathrm{m}$. At lower $p_{t}$ the main contribution to the impact parameter resolution is the material budget although the ITS can still track particles down to $80 \mathrm{MeV} / \mathrm{c}$.

The ITS allows also the identification of low momentum particles by mean of the $\mathrm{dE} / \mathrm{dx}$ mea- 

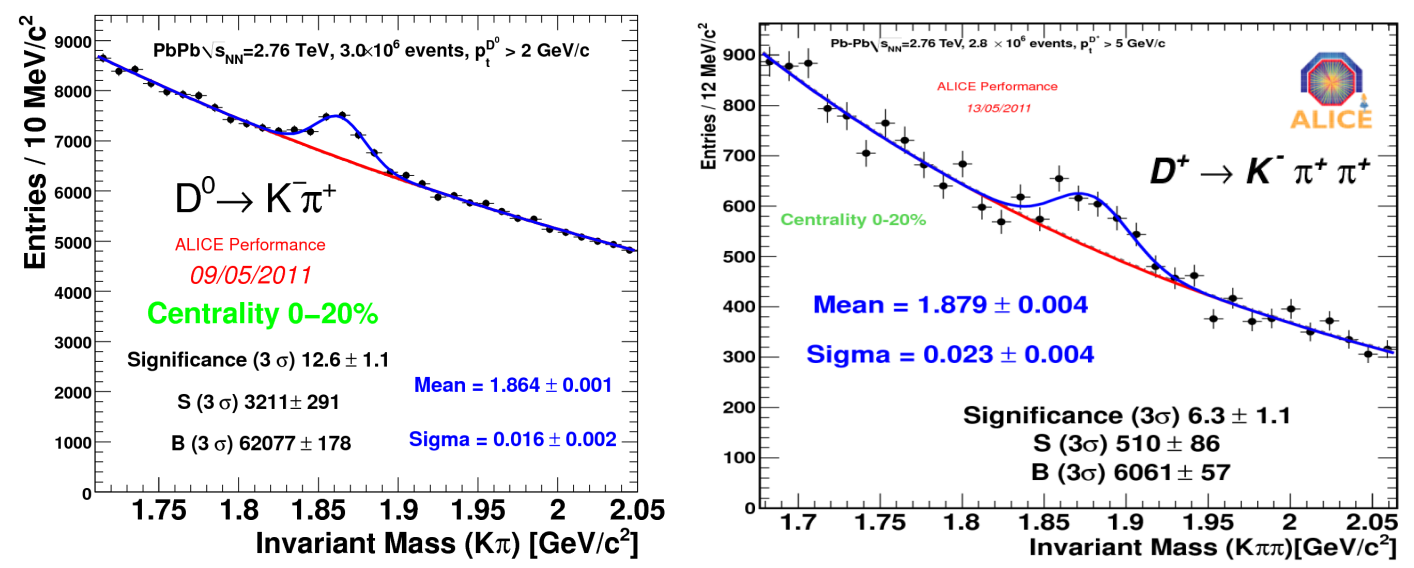

Figure 4: Invariant mass distribution of $D^{0} \rightarrow K^{-} \pi^{+}$candidates with $p_{t}>2 \mathrm{GeV} / \mathrm{c}$ (left) and $D^{+} \rightarrow K^{-} \pi^{+} \pi^{+}$ candidates with $p_{t}>5 \mathrm{GeV} / \mathrm{c}$ (right) in the $20 \%$ most central $\mathrm{Pb}-\mathrm{Pb}$ collisions at $\sqrt{s_{N N}} 2.76 \mathrm{TeV}$.

surement in the non-relativistic region. This measurement is based on the analogue information proportional to the charge collected by the outermost layers of the ITS, i.e. SDD and SSD. The Energy loss $(\mathrm{dE} / \mathrm{dx})$ in each layer is corrected for the particle path length inside the silicon. The truncated mean method is applied to account for the long tails of the Landau distribution. The p-p and $\mathrm{Pb}-\mathrm{Pb}$ results are shown in figure 3. The ITS capability provides pions/kaons separation up to $0.5 \mathrm{GeV} / \mathrm{c}$ and pions/protons separation up to $1 \mathrm{GeV} / \mathrm{c}$.

The precise measurement of the track impact parameter and the capability to separate the secondary vertices originating from heavy flavour decays are fundamental requirements for the heavy flavour physics. The analysis strategy for the study of charmed mesons is based on the invariant mass analysis of fully reconstructed decay topologies originating from displaced vertices. The aim is to correctly assign the tracks belonging to the open charm decays from the full sample of reconstructed tracks. Due to the large combinatorial background, a series of selection criteria has been defined: the cuts are optimized to maximize the statistical significance of each specific decay signal. The particle identification is also applied for the track selection, in particular in the high multiplicity environment of $\mathrm{Pb}-\mathrm{Pb}$ collisions. As an example of the good performance achieved with the ITS, the invariant mass distributions of two benchmark channels, namely $D^{0} \rightarrow K^{-} \pi^{+}$ and $D^{+} \rightarrow K^{-} \pi^{+} \pi^{+}$, in $\mathrm{Pb}-\mathrm{Pb}$ central collisions are shown in figure 4.

\section{Upgrade plans}

In the previous sections it was shown that the ITS is performing according to the design specifications. However the technologies used to build it are already ten years old and impressive progress has happened in the field of semiconductor detectors for tracking in high energy physics experiments. Furthermore it is reasonable to assume that the lifetime of a detector like the ITS, even if the ALICE environment is not as demanding as the other LHC experiments, cannot be longer than ten years. So far there are no striking evidences yet of aging and the fraction of the detectors that cannot be operated can be actually traced to instrumental defects. Taking into account the current detector technologies and the knowledge of the LHC operation, the ITS could be redesigned in 

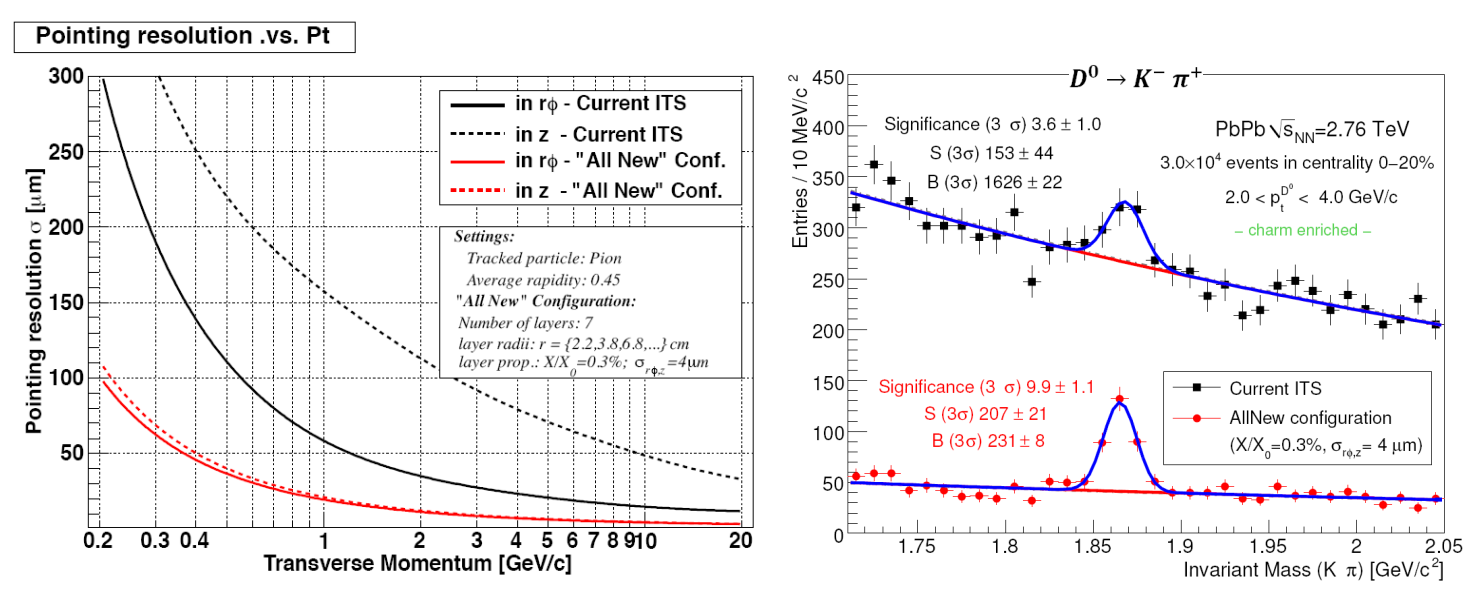

Figure 5: The left plot shows the impact parameter resolution as a function of the momentum for the current ITS (black curves) and one of the layout option under study for the ITS upgrade (red curves) both in the r $\phi$ and z plane. The right plot shows the invariant mass distribution of $D^{0} \rightarrow K^{-} \pi^{+}$candidate with $\left.2.0<p_{t}\right\rangle$ 4.0 GeV/c simulated in central $\mathrm{Pb}-\mathrm{Pb}$ collisions for the current ITS and the upgraded layout.

order to extend the physics capabilities of ALICE. The ongoing developments on semiconductor detectors allow the design of a silicon tracker even lighter than the current ITS. Moreover, the impressive progress made by the accelerator team in running LHC allows to conceive, after only two years of operation, a substantial reduction of the beam pipe diameter. This option would allow to bring the first detector layer closer to the interaction point. Based on the above considerations, an intensive study has been recently started for upgrading the ITS [15]. A lighter innermost layer closer to the interaction point would allow a substantial improvement of the track impact parameter resolution extending the ALICE heavy flavour physics capabilities in heavy-ion collisions also at very low $p_{t}$. The heavy flavour baryons, as for instance the $\Lambda_{c}$, could also be studied thanks to the improved signal-to-background ratio.

Several scenarios are being evaluated for an upgrade of the ITS with the aim to improve the impact parameter resolution by a factor 2 or 3 in the bending plane and by a factor 10 along the beam axis. This can be achieved placing the first pixel layer at $\approx 23 \mathrm{~mm}$ instead of the actual $39 \mathrm{~mm}$ and reducing the dimension of the pixel cell from the current $50 \times 425 \mu \mathrm{m}^{2}$ to $20 \times 20 \mu \mathrm{m}^{2}$. The material budget should also be reduced to $0.3 \div 0.5 \% X / X_{0}$ per layer with respect to the current $1.14 \% X / X_{0}$ in order to improve the tracking capability at very low momentum. The possibility to extend the trigger capability to identify displaced vertices within the L2 trigger latency is also under discussion.

The figure 5 (left) shows the pointing resolution as a function of $p_{t}$ simulated for one of the proposed solution of the new ITS (red curves) compared with the existing ITS (black curves) both in $\mathrm{r} \phi$ and z. The layout assumed in the simulation is a complete new ITS made of 7 layers with a $4 \mu \mathrm{m}$ spatial precision and material budget of $0.3 \%$ of $X / X_{0}$ per layer. On the right side of the same figure the effect of the new layout on the $D^{0} \rightarrow K^{-} \pi^{+}$signal significance is shown. The $D^{0}$ invariant mass distribution in $p_{t}$ range $2-4 \mathrm{GeV} / \mathrm{c}$ in central $\mathrm{Pb}-\mathrm{Pb}$ collisions is compared with the current ITS performance. A substantial reduction of the background is visible thus improving the signal-to-background ratio by almost a factor three. 


\subsection{Conclusions}

After the commissioning phase, the ITS detector is performing according to the design values and is providing a reliable contribution to the ALICE tracking and vertex reconstruction in all LHC configurations, with proton and lead beams. Moreover, the ITS is contributing with the SPD to the trigger decision and with the SDD and SSD to the particle identification. Some local inefficiencies have been detected and the list of actions to be taken during the scheduled shutdown in 2013 is being elaborated. The study to upgrade the ITS is ongoing with the aim to extend the physics capabilities of ALICE in particular for the heavy flavours.

\section{References}

[1] ALICE Collaboration, ALICE: Physics Performance Report, Volume I, J. Phys., G30 (2004), 1517-1763

ALICE Collaboration, ALICE: Physics Performance Report, Volume II, J. Phys., G32 (2006), $1295-2040$

[2] ALICE Collaboration, The ALICE experiment at the CERN LHC, JINST, 3 (08) S08002

[3] G. Aglieri Rinella et al., The pixel trigger system for the ALICE experiment PoS (RD09) 005 (2009)

[4] R. Santoro et al., The ALICE Silicon Pixel Detector: readiness for the first proton beam JINST, 4 P03023 (2009)

[5] V.Manzari, The Alice Inner Tracking System: commissioning and running experience, PoS (Vertex 2009) 005 (2009)

[6] M.Sitta and P.Christakoglou, Operations and performance of the Silicon Drift and Silicon Strip Detectors of the ALICE experiment, PoS (Vertex 2010) 009 (2010)

[7] R. Turrisi et al., ALICE Pixel Detector operations and performance PoS (VERTEX 2010) 007 (2010)

[8] C. Cavicchioli et al., Calibration of the Prompt LO Trigger of the Silicon Pixel Detector for the ALICE Experiment, Proceedings of TWEPP 2009, Paris, France, Sep. 2009

[9] F. Prino et al., Characterization of the ALICE Silicon Drift Detectors using an infrared laser, JINST, 3 P06004 (2008)

[10] ALICE Collaboration, First proton - proton collisions at the LHC as observed with the ALICE detector: measurement of the charged-particle pseudorapidity density at $\sqrt{s}=900 \mathrm{GeV}$, Eur.Phys.J.C, $\mathbf{6 5}$ (2010), 111-125

[11] ALICE Collaboration, Alignment of the ALICE Inner Tracking System with cosmic-ray tracks, JINST, 5 P03003 (2010)

[12] C. Bombonati et al., Alignment of the ALICE Inner Tracking System with cosmic-ray tracks, ALICE-INT-2009-035 (2009)

A. Dainese et al., Validation of the survey measurement for the alignment of the Silicon Strip Detector using cosmic-ray tracks, ALICE-INT-2009-045 (2009)

[13] B. Batyunya, Yu. Belikov, K. Safarik, ALICE Internal Note, ALICE-INT-97-24 (1997)

[14] E. Crescio et al., Performance of the ITS stand-alone tracker in pp collisions, ALICE-INT-2009-046 (2009)

[15] ALICE ITS Upgrade Collaboration, Alice ITS Upgrade - Conceptual Design Report (CDR), In preparation 